\title{
The Effectiveness of an AR-based Context-Aware Assembly Support System in Object Assembly
}

\author{
Bui Minh Khuong* \\ Osaka University
}

\author{
Kiyoshi Kiyokawa \\ Osaka University \\ Tomohiro Mashitall \\ Osaka University
}

\author{
Andrew Miller \\ University of Maryland \\ Haruo Takemurall \\ Osaka University \\ Joseph J. LaViola Jr.
University of Central Florida \\ Joseph J. LaViola Jr.
University of Central Florida
}

\begin{abstract}
This study evaluates the effectiveness of an AR-based contextaware assembly support system with AR visualization modes proposed in object assembly. Although many AR-based assembly support systems have been proposed, few keep track of the assembly status in real-time and automatically recognize error and completion states at each step. Naturally, the effectiveness of such contextaware systems remains unexplored. Our test-bed system displays guidance information and error detection information corresponding to the recognized assembly status in the context of building block (LEGO) assembly. A user wearing a head mounted display (HMD) can intuitively build a building block structure on a table by visually confirming correct and incorrect blocks and locating where to attach new blocks. We proposed two AR visualization modes, one of them that displays guidance information directly overlaid on the physical model, and another one in which guidance information is rendered on a virtual model adjacent to the real model. An evaluation was conducted to comparatively evaluate these AR visualization modes as well as determine the effectiveness of context-aware error detection. Our experimental results indicate the visualization mode that shows target status next to real objects of concern outperforms the traditional direct overlay under moderate registration accuracy and marker-based tracking.
\end{abstract}

Index Terms: H.5.1 [Information Interfaces and Presentation]: Multimedia Information Systems-Artificial, augmented, and virtual realities; H.5.1 [Information Interfaces and Presentation]: Multimedia Information Systems-Evaluation/methodology

\section{INTRODUCTION}

One of the most promising applications of AR (i.e., systems that blend computer generated virtual objects with the real environment [3]) is in the traditional manufacturing assembly domain. In manufacturing, while some assembly operations are automated, there are still a significant number of assembly operations that require manual human effort. For example, in automobile assembly, the fabrication of body and chassis are typically automated, while the final assembly of interiors, trim, and lighting are manual. Manual assembly is also common in manufacturing processes where automation is not cost-effective, products are highly customized, or processes cannot be done by automatic machines (e.g., high quality soldering).

\footnotetext{
*e-mail: bmkhuong@lab.ime.cmc.osaka-u.ac.jp

†e-mail:kiyo@ime.cmc.osaka-u.ac.jp

†e-mail:amiller@cs.umd.edu

§e-mail:jj1@eecs.ucf.edu

IIe-mail:mashita@ime.cmc.osaka-u.ac.jp

"e-mail:takemura@ime.cmc.osaka-u.ac.jp
}

There are several reasons why AR may improve this process of manual assembly. AR technology makes it possible to display digital information in the assembly subject's field of view, such as step-by-step instructions, 3D illustrations, or other data that are essential for the work. In comparison to conventional systems such as paper based work instructions or multimedia information systems, information in AR applications can be displayed depending on the context (i.e., in reference to particular components or subassemblies). The context-related visualization of information helps to reduce search time in assembly. In addition, secondary operations such as reading textual information can be reduced. AR also makes it possible to reduce head and eye movements [10] and the cost of attention switching as well as improve spatial perception to increase productivity [17][4]. Finally, AR has the potential to resolve spatial ambiguities by displaying spatial indicators (such as arrows or spotlights) properly registered and directly overlaying the actual workpiece, freeing the user from the cognitive burden of relating actual locations on the workpiece to corresponding locations on a separate virtual model [16].

Although a number of assembly support system prototypes and test-bed applications using AR have been proposed since the early 1990s [21], they are mainly proof-of-concept applications, and are limited particularly in the ways that the user can interact with the workpiece. Recently, however, context-aware on-line error detecting AR systems have attracted attention [14]. This enables us to evaluate the potential of augmented reality in a more comprehensive way.

The purpose of this study is to evaluate the effectiveness of a test-bed AR-based context-aware assembly support system in two $\mathrm{AR}$ visualization modes in order to understand how best to assist users in the assembly context.

\section{Related Work}

One of the most well-known applications of AR in the assembly domain is the assembly of cable harnesses at Boeing [3]. Their augmented reality project was designed to display pertinent instructions and diagrams in front of manufacturing workers, who use the information to work on or assemble pieces of the aircraft. A small, head-mounted video camera detects visual markers on the workpiece and the computer estimates the relative pose information and displays the diagram and text on the workpiece. The STARMATE project [19], aims at specifying, designing, developing, and demonstrating a prototype allowing computer guided maintenance of complex mechanical elements using augmented reality techniques. In the STARMATE system, a user equipped with a see-through head mounted display (HMD), a microphone and headphones, has to perform tasks on a mechanical element. The ARVIKA project [1] uses augmented reality technologies to research and implement useroriented and application driven support solutions for a variety of working procedures. The workflow control is implemented using speech control. The tracking is based on recognition of visual fiducial markers. The AR systems are linked to HTML-based bills of material and work schedule plans. Another project, ARTESAS 
(Advanced Augmented Reality Technologies for Industrial Service Applications) [2] aims to provide augmented reality systems to be used in complex industrial service situations. As a follow-up to the ARVIKA project, the main goal in the ARTESAS project is to replace the marker-based tracking system with a marker-less one.

Although new solutions and techniques for building up an innovative assembly support system have been proposed and improved through the years, there are still limitations specifically in object tracking and recognition techniques that need to be overcome. Focusing on the assembled objects themselves, a context-aware assembly support system that keeps track of the objects' status in realtime, automatically recognizing error and completion states at each assembly step and displaying guidance information corresponding to the recognized states, is desirable and is expected to meet the needs for good training, improving labor efficiency and accuracy in work.

Recently, there has also been work to track and evaluate the correctness of assembly steps presented to the user. Molineros et al. [15] put encoded markers on each part for tracking it and detecting connections with other parts. Gupta et al. [9] present a real-time system which infers and tracks the assembly process of a building block model. The system makes it possible to build a virtual replica of the model at every step, enabling users to author a new model and use the inferred assembly process to guide its recreation by others. It is also able to detect mistakes made and helps correct them by providing appropriate feedback. They propose a way of assembly guidance where the next blocks to be added is rendered in blinking mode with the tracked virtual model on screen. However, in their system, they require users to put blocks being added or removed in designated Add or Remove boxes on the table surface to let the system infer a virtual replica of the blocks at every step. This reduces naturalness of assembly operations. Furthermore, in their system, there exists a perception transfer problem that can occur by displaying assembly guidance on a monitor screen. In comparison, our test-bed system can make it possible for users to add or remove parts of a model being assembled naturally, since it automatically recognizes parts added to or removed from the model and updates the virtual replica of the model in real-time in the form of AR.

There has also been a rich body of work on AR visualization methods. AR information needs to be presented to the user in such a way that ambiguities are minimized as to what the information is referring to. Most studies on AR visualization methods cope with two typical sources of such ambiguities; misinterpretation of depth orders and registration errors. For the former problem, it is well known that AR information with solid rendering appears to be frontmost regardless of its intended depth. To better convey spatial relationships to the real objects, AR information is often rendered with a cut away box or in a semi-transparent manner [8][5]. A combination of wireframe and semi-transparent rendering is proven to help discern depth ordering [13]. For the latter problem, in the presence of registration error, expanded boundary regions based on estimated registration errors have been proposed to disambiguate the target object of concern [6][7]. Robertson et al. report additional visual context can also ameliorate the negative effects of registration error [18].

In our work, we focus on AR visualization modes of assembly guidance as well as evaluating them to explore AR visualization methods to best assist users in assembly tasks. There has also been work to explore the effectiveness of AR as well as AR visualization methods in the assembly, maintenance and repair domains. Henderson et al. [11] explore how real-time computer graphics, overlaid on and registered with the actual repaired equipment, can improve the productivity, accuracy, and safety of maintenance personnel. Their user study shows that participants were able to complete psychomotor aspects of the assembly task significantly faster and with significantly greater accuracy with AR than when using 3D-

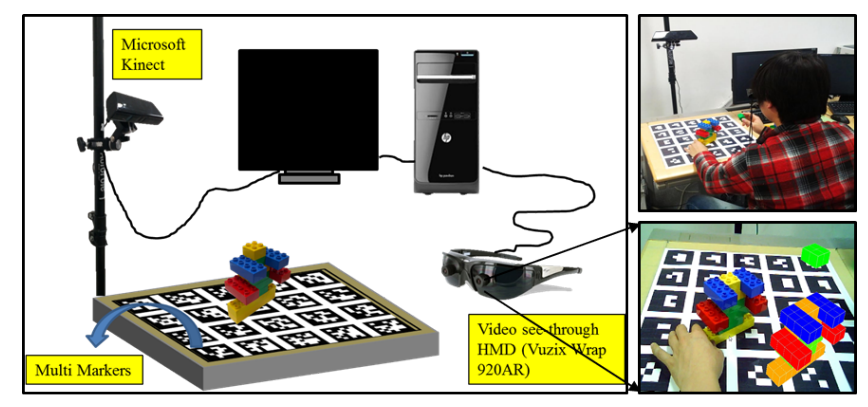

Figure 1: The prototype system for guided assembly of building block (LEGO) structures.

graphics-based assistance presented on a stationary LCD. Qualitative questionnaire results indicate that participants overwhelmingly preferred the AR condition, and ranked it as more intuitive than the LCD condition. Tang et al. [20] describe an experiment that compared the effectiveness of AR instructions against three other types of instructional media (a printed manual, computer assisted instruction (CAI) using a monitor-based display, and CAI utilizing a head-mounted display. The result of the experiment shows that overlaying 3D instructions (AR instructions) on the actual workpieces reduced the error rate for an assembly task by $82 \%$, particularly diminishing cumulative errors. These studies validated the effectiveness of AR-assisted operations over traditional monitorbased or paper-based operations. In contrast to this prior work, we investigate how AR-assisted operations can gain further benefit from automatic error detection and completion recognition during the assembly process. Also, we explore another visualization technique (in which guidance information is rendered on a virtual model adjacent to the real model) which does function well under poor geometric registration, that is different from the traditional direct overlay.

\section{The Test-Bed System}

The hardware setup of the test-bed system is shown in Figure 1. The user builds a building block structure from the table up, layer by layer, while the system highlights the next layer to build on the virtual representation of the real model being assembled. The system uses depth information captured in real-time by a depth sensor, the Microsoft Kinect, for acquiring and tracking. A video see-through head mounted display (Vuzix Wrap 920AR) is used to display assembly guidance information to the user. Due to limitations of the alignment and field-of-view of this hardware display, we chose to present guidance in the form of non-stereo biocular imagery.

\subsection{D Model Construction and Tracking}

A depth camera-based system using an algorithm called LatticeFirst [14] is used for real-time acquisition and tracking of LEGO blocks. It is fast and effective, providing users with the ability to incrementally construct a block-based physical model using their bare hands while the system updates the model's virtual representation in real-time. However, the physical model must be rotated around $360^{\circ}$ or more for full reconstruction because the tracking system uses only one Kinect suspended about 0.5 meter above the table on a stand opposite the user. If the models are not rotated, the test-bed system has no line of sight to see occluded parts of the assembly. Due to filtering in the tracking system, there is slight but noticeable latency (up to approximately one tenth of a second) such that when the physical model is moving, the virtual model appears to lag behind. 


\subsection{Assembly Guidance and Error Detection}

The virtual representation of the physical model being assembled is updated and compared with the target model of the same size in a $3 \mathrm{D}$ voxel grid space in real-time. The system estimates model geometry based on estimating occupied voxels on each model. Two models are compared in real-time based on occupied voxels information estimated to find out parts that should be filled or be marked as error in the physical model (see Figures 2 and 3).

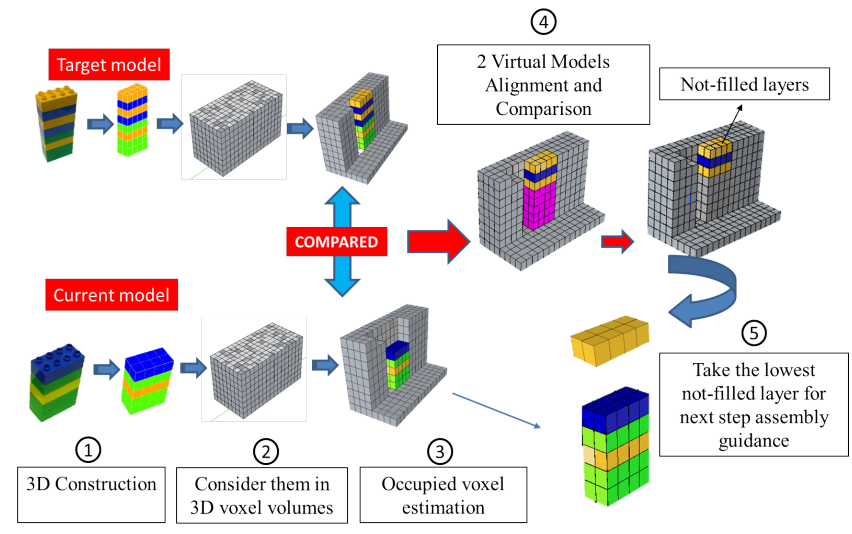

Figure 2: Assembly guidance mechanism. Two models are compared in real-time based on occupied voxels information estimated in every frame. The lowest layer of parts that have not been filled in is displayed as the next assembly step.

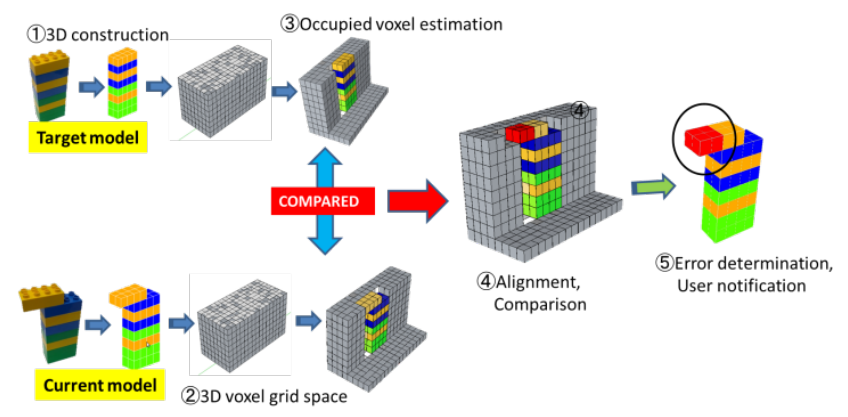

Figure 3: Error detection mechanism. Blocks that exist in the physical model but do not exist in the target model are marked as error blocks.

\section{Visualization Modes}

We propose two context-related visualization modes (see Figure 4) for an AR-based context-aware assembly support system . In the first mode (Figure 4a), the assembly instructions (the next blocks to add) are displayed as 3D animated wireframe blocks, directly overlaying the physical model. The blocks are animated with a downward motion that suggests snapping the block in place. A partial wireframe containing just the topmost layer of the virtual model is additionally rendered overlaying the actual model. The display of the partial wireframe has the potential to reduce spatial ambiguity by overlaying instructions directly on the real model while it also helps users to figure out the correct position of next blocks in poor alignment situations. In the second mode (Figure 4b), the assembly instructions are displayed on top of a separate virtual model that is rendered to the side of the actual model, but in the same orientation. Pilot studies indicate that each of the two modes is better in task completion time and user preference than a naive mode where a full wireframe representation of the virtual model is rendered overlaying the actual model.

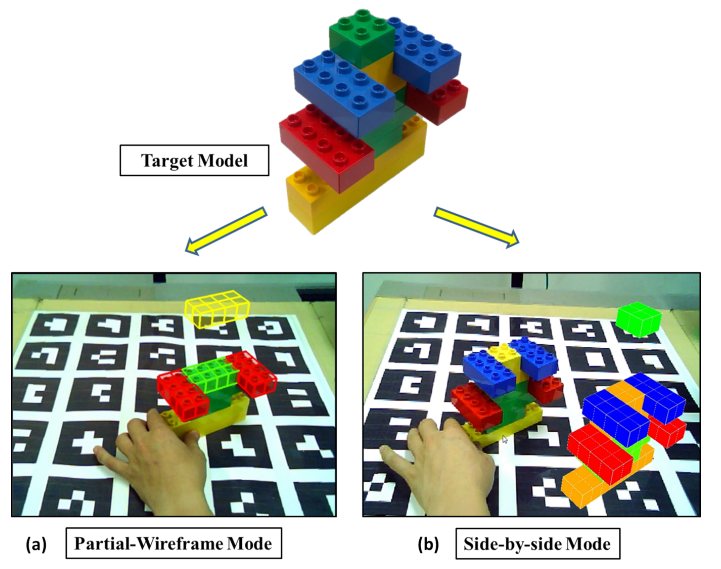

Figure 4: Proposed visualization modes.

\subsection{Evaluation}

The primary objective of this evaluation is to compare the two context-related visualization modes proposed (see Figure 4), in order to provide design suggestions about how to best assist users in assembly tasks. A secondary goal of the evaluation is to determine the effectiveness of context-aware error detection. We modified the test-bed so that the error detection mechanism could be enabled or disabled, and used this as a second independent variable.

\subsubsection{Hypothesis}

With error detection enabled, the user needs to rotate the model and the system will automatically detect completion status as well as errors and notify the user in real-time. With error detection disabled, users do not need to rotate the model. Instead, they can use a left mouse button to forward assembly steps and right mouse button to backward assembly steps. However, users have to check and determine completion state as well as errors at each step by themselves. The first visualization mode (called Partial-wireframe overlay mode) can have problems with misalignment and users may need to pay more attention and time to determine the right position of blocks carefully. The second visualization mode (called Sideby-side mode) with a colorful, solid (not $80 \%$ transparent as in the Partial-wireframe overlay mode) virtual representation displayed beside the real models not only avoids effects of misalignment but also makes it easier for users to see and determine the next guided blocks position. So, this mode can help users shorten the completion time, lower stress to finish the models and should expect the Side-by-side mode to be useful in assembly tasks.

We therefore make the following predictions:

- MH1: The Partial-wireframe overlay mode will not achieve better completion time of assembly tasks when compared to the Side-by-side mode.

- MH2: Visualization modes without error detection will achieve better completion time of assembly tasks but achieve higher error rates when compared to visualizations modes with support of error detection.

- MH3: When compared to the Partial-wireframe overlay mode, the Side-by-side mode is significantly better in aspects. ease of understanding, ease of seeing, stress level, familiarity, satisfaction level and usefulness.

\subsubsection{Subjects}

We recruited 24 people (12 males, 12 females) from many different departments of Osaka University for participation in this evalua- 


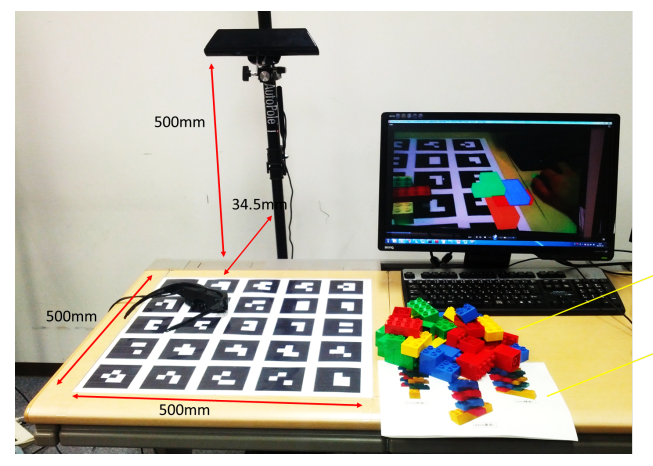

Figure 5: System configuration for the evaluation.
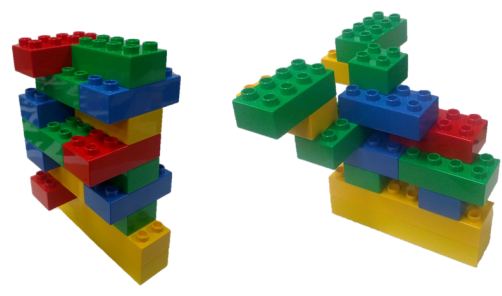

Figure 6: Assembly task models for the evaluation.

tion. They are from five countries in three continents. The ages of participants were between 22 and 35 years. Only 5 participants had prior experience with AR. None of the participants had used any AR assembly support system before. Sixteen participants reported that this is the first time they used LEGO or Duplo blocks.

\subsubsection{Apparatus}

Our evaluation was conducted on a desktop computer with an Intel core 2 Duo E7400 2.80GHzx2 processor, an NVIDIA (B) GeForce (R)T230 GPU and three gigabytes of memory. A Microsoft Kinect was used as a depth sensor, and a video see-through head mounted display (HMD) - the Vuzix Wrap 920AR - was used for displaying visual feedback (see Figure 5).

\subsubsection{Experiment Design and Procedure}

We used a $2 \times 2$ factorial within-subjects experimental design. The independent variables were the visualization mode (called VIS factor) for presenting assembly instructions (the Side-by-side mode or the Partial-wireframe overlay mode) and whether or not error detection was enabled (called ERR factor). The dependent variables were the time taken to complete the task, number of errors, ease of use, ease of understanding, ease of seeing, stress level, familiarity, satisfaction, and usefulness. All twenty-four participants were subject to all four conditions in a randomized order. For each condition, the participants were asked to assemble two building block (Duplo) models in randomized order (see Figure 6). We abbreviate the four conditions as follows: S_ON: the Side-by-side mode with error detection, S_OFF: the Side-by-side mode without error detection, PW_ON: the Partial-wireframe overlay mode with error detection and PW_OFF: the Partial-wireframe overlay mode without error detection.

The completion time, number of errors made during assembly, and number of errors present in the completed model were recorded for each task. After finishing each assembly task, each participant was asked to fill out a questionnaire asking them for feedback about their experience with each condition using the questions shown in Table 1. The questionnaire consisted of 7-point Likert scale responses, with 1 indicating the most negative response and 7 indicating the most positive response.
Table 1: Questionnaire for evaluating the effectiveness of conditions.

\begin{tabular}{|c|c|c|}
\hline No & Question & Response Type \\
\hline 1 & $\begin{array}{l}\text { Were the assembly instructions } \\
\text { information and error notifica- } \\
\text { tion difficult to understand? }\end{array}$ & $\begin{array}{l}\text { 7-point Likert } \\
\text { (1:Difficult to un- } \\
\text { derstand; 7:Easy to } \\
\text { understand) }\end{array}$ \\
\hline 2 & $\begin{array}{l}\text { Were the assembly instructions } \\
\text { information and error notifica- } \\
\text { tion difficult to see? }\end{array}$ & $\begin{array}{l}\text { 7-point Likert } \\
\text { (1:Difficult to see; } \\
\text { 7:Easy to see) }\end{array}$ \\
\hline 3 & $\begin{array}{l}\text { Did you feel stress when using } \\
\text { this assembly instructions me- } \\
\text { dia? }\end{array}$ & $\begin{array}{l}\text { 7-point Likert } \\
\text { (1:Feel very } \\
\text { stressed; 7:Do not } \\
\text { feel the stress) }\end{array}$ \\
\hline 4 & $\begin{array}{l}\text { Did you feel difficult to become } \\
\text { familiar with the assembly in- } \\
\text { structions media? }\end{array}$ & $\begin{array}{l}\text { 7-point Likert } \\
\text { (1:Difficult to } \\
\text { become familiar; } \\
\text { 7:Easy to become } \\
\text { familiar) }\end{array}$ \\
\hline 5 & $\begin{array}{l}\text { Did you feel satisfied with the } \\
\text { assembly instructions media af- } \\
\text { ter using it? }\end{array}$ & $\begin{array}{l}\text { 7-point Likert } \\
\text { (1:Not satisfied at } \\
\text { all; 7:Very satis- } \\
\text { fied) }\end{array}$ \\
\hline 6 & $\begin{array}{l}\text { Did you feel the assembly in- } \\
\text { structions media useful for the } \\
\text { assembly tasks? }\end{array}$ & $\begin{array}{l}\text { 7-point Likert } \\
\text { (1:Not useful at all; } \\
\text { 7:Very useful) }\end{array}$ \\
\hline
\end{tabular}

\subsubsection{Analysis of quantitative data}

Figures 7, 8 and 9 illustrate respectively the mean time of completion, errors made during the task, and errors present in the completed model. Stars indicate significance levels as follows: * $\mathrm{p}<0.05$, ** $\mathrm{p}<0.01$, *** $\mathrm{p}<0.001$. The Side-by-side mode resulted in a shorter completion time than Partial-wireframe mode, irrespective of the error detection mechanism. Among the conditions, S_OFF had the shortest time of completion while PW_OFF had the longest. Subjects using the Partial-wireframe overlay mode made more errors than with the Side-by-side mode. Without error detection, participants rarely noticed errors they made during the assembly process; however, with error detection enabled, participants recognized and fixed all errors by themselves during the assembly process.

We conducted a repeated measure ANOVA test among these conditions. In mean completion time, we found significance for VIS factor $\left(F_{1,23}=27.311, \mathrm{p}<0.0001, \eta_{p}^{2}=0.543, \mathrm{OP}=0.999\right)$ and ERR factor $\left(F_{1,23}=7.03, \mathrm{p}<0.05, \eta_{p}^{2}=0.234\right.$, OP $\left.=0.719\right)$ but did not find significance for the interaction between VIS and ERR (VISxERR) $\left(F_{1,23}=0.102, \mathrm{p}=0.752, \eta_{p}^{2}=0.004\right.$, OP $\left.=0.061\right)$. In error rate during assembly, we found significance for VIS factor $\left(F_{1,23}=9.364, \mathrm{p}<0.006, \eta_{p}^{2}=0.289, \mathrm{OP}=0.834\right)$, but did not find significance for ERR factor $\left(F_{1,23}=0.276, \mathrm{p}=0.605, \eta_{p}^{2}=0.012\right.$, $\mathrm{OP}=0.080)$ and VISxERR $\left(F_{1,23}=0.284, \mathrm{p}=0.599, \eta_{p}^{2}=0.012\right.$, $\mathrm{OP}=0.080)$. In error rate after completion, we found significance for VIS factor and ERR factor $\left(F_{1,23}=4.713, \mathrm{p}<0.05, \eta_{p}^{2}=0.170\right.$, $\mathrm{OP}=0.548)$ and $\left(F_{1,23}=14.57, \mathrm{p}<0.001, \eta_{p}^{2}=0.388, \mathrm{OP}=0.955\right)$ as well as the interaction between them $\left(F_{1,23}=4.173, \mathrm{p}<0.05, \eta_{p}^{2}\right.$ $=0.0170, \mathrm{OP}=0.548)$. We ran post-hoc analyses using pairwise t-tests with the Holm's Bonferroni adjustment to isolate the significant differences [12]. We found that the mean completion time for S_OFF was significantly faster than S_ON $\left(t_{23}=1.426, \mathrm{p}<0.05\right)$, and that each of these modes were significantly faster than either PW_ON (S_ON: $t_{23}=-3.415, \mathrm{p}<0.0125$; S_OFF: $t_{23}=-5.176, \mathrm{p}$ $<0.0083$ ) or PW_OFF (S_ON: $t_{23}=-2.460$, p $<0.025$; S_OFF: $t_{23}$ $=-4.773, \mathrm{p}<0.01)$. We found significant differences in the error 


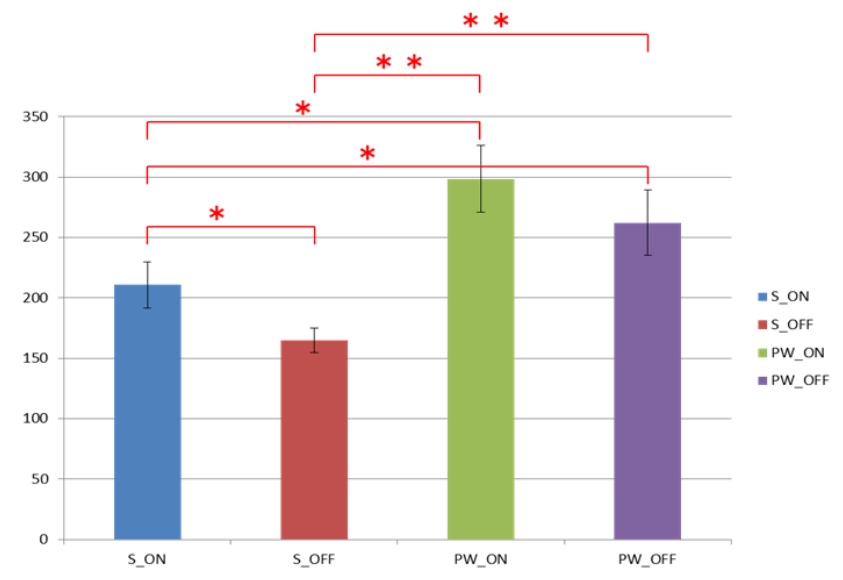

Figure 7: The mean of completion time of each condition in the evaluation (the second unit). Error bars indicate $95 \%$ confidence intervals.

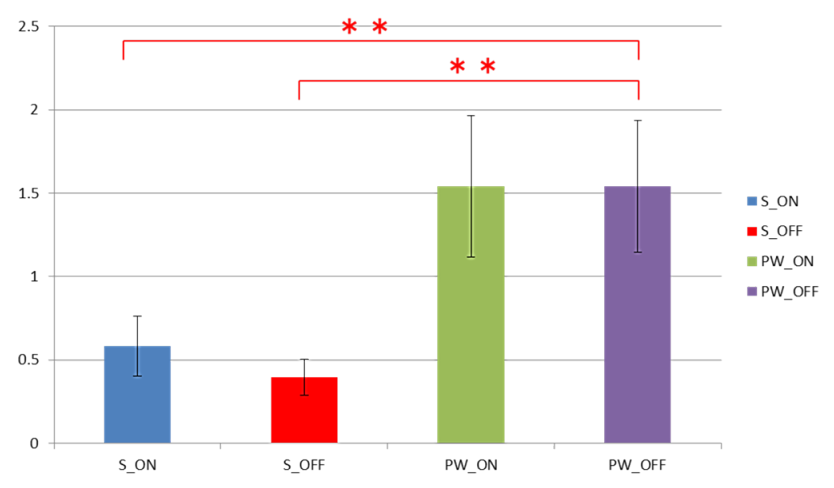

Figure 8: Mean number of errors per assembly task of each condition during the assembly process. Error bars indicate $95 \%$ confidence intervals.

rate during assembly between the S_ON and the PW_OFF conditions $\left(t_{23}=-2.932, \mathrm{p}<0.0083\right)$ and between the PW_ON and the PW_OFF conditions $\left(t_{23}=-2.916, \mathrm{p}<0.01\right)$. For error rate after completion we found significant differences between S_ON and the PW_OFF $\left(t_{23}=-3.317, \mathrm{p}<0.0083\right)$ and between the PW_ON and the PW_OFF $\left(t_{23}=-3.137, \mathrm{p}<0.01\right)$. Due to the Holm's Bonferroni adjustment, the differences between S_ON and the S_OFF $\left(t_{23}\right.$ $=-2.015, \mathrm{p}=0.056)$ and between the PW_ON and the S_OFF $\left(t_{23}\right.$ $=-2.015, \mathrm{p}=0.056)$ were not found to be significant; however, the small $p_{-}$value suggests that these differences may be significant with more participants.

\subsubsection{Analysis of questionnaire data}

Figure 10 shows the ranking that participants provided for each condition in each aspect: ease of understanding, ease of seeing, stress level, familiarity, satisfaction level and usefulness.

Based on the rankings elicited from the participants, the Sideby-side mode had better performance than Partial-wireframe overlay mode in every qualitative aspect (see Table 2). We used a nonparametric Friedman test to check for significant differences in each qualitative metric among the conditions, followed by Wilcoxon signed rank tests.

\section{Discussion}

In the evaluation, we found that the Side-by-side mode had the shorter completion time than the Partial-wireframe overlay mode regardless of the presence of error detection. This finding supported

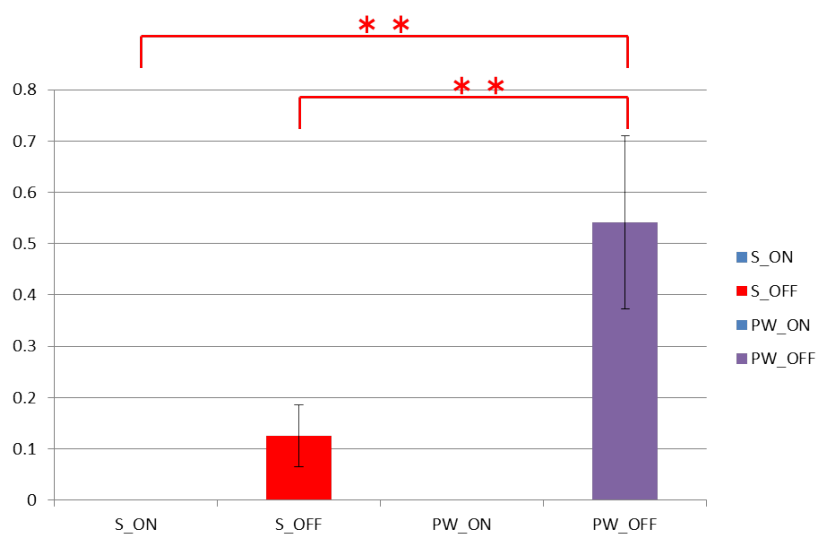

Figure 9: Mean number of errors per assembly task found on models after completing the assembly task. Error bars indicate $95 \%$ confidence intervals.

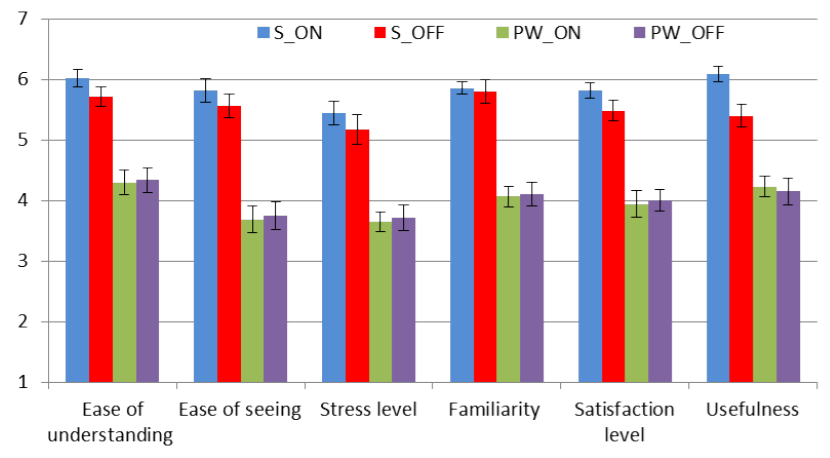

Figure 10: Conditions ranked on usefulness level in the evaluation.

our hypothesis (MH1). Participants reported that because of misalignment and the potential to mistake some parts of the real model for parts of the virtual representation of the same color, they spent more time trying to determine the position of next blocks as they did in the Side-by-side mode. It may still be the case that AR has the potential to reduce spatial ambiguity by overlaying instructions directly on the real model; however it seems that this is highly sensitive to misalignment, latency, or conflicting depth cues. At least for our test-bed, having a spatial separation between the virtual model and the real model led to significantly better performance in every aspect (MH3).

The result of this evaluation also showed that S_OFF (the Sideby-side mode without error detection) had shorter completion time than S_ON (the Side-by-side mode with error detection) and this difference was significant. On the other hand, S_OFF had higher error rates than $\mathrm{S}_{-} \mathrm{ON}$ after assembly finished $(\mathrm{p}=0.056)$. Although the difference was not found to be significant due to the Holm's Bonferroni adjustment, with the small p-values we believe it may be significant with more participants and more complex models. Hypothesis (MH2) was therefore only partially supported.

\section{FUTURE WORK}

For future work, there are some improvements that could be made. First, the overlay mode needs to be improved to have a better alignment between assembly instructions and physical models. Modelbased tracking and / or external sensors attached directly on blocks could be considered. Second, an optical see-through head mounted display (HMD) should be tested as an alternative to the video seethrough HMD used in the test-bed system. Although the cur- 
Table 2: Significant results from analysis of questionnaire data.

\begin{tabular}{r|r|r|r|r|r}
\hline & Friedman & S_ON $>$ PW_ON & S_ON $>$ PW_OFF & S_OFF $>$ PW_ON & S_OFF $>$ PW_OFF \\
\hline Ease of understanding & $X^{2}=43.590, \mathrm{p}<0.0001$ & $\mathrm{z}=4.311, \mathrm{p}<0.0083$ & $\mathrm{z}=3.999, \mathrm{p}<0.01$ & $\mathrm{z}=3.519, \mathrm{p}<0.0125$ & $\mathrm{z}=3.646, \mathrm{p}<0.0167$ \\
Ease of seeing & $X^{2}=45.069, \mathrm{p}<0.0001$ & $\mathrm{z}=3.921, \mathrm{p}<0.0083$ & $\mathrm{z}=4.141, \mathrm{p}<0.01$ & $\mathrm{z}=3.928, \mathrm{p}<0.0125$ & $\mathrm{z}=3.714, \mathrm{p}<0.0167$ \\
Stress level & $X^{2}=48.433, \mathrm{p}<0.0001$ & $\mathrm{z}=4.120, \mathrm{p}<0.0083$ & $\mathrm{z}=4.026, \mathrm{p}<0.01$ & $\mathrm{z}=3.652, \mathrm{p}<0.0125$ & $\mathrm{z}=3.839, \mathrm{p}<0.0167$ \\
Familiarity & $X^{2}=54.041, \mathrm{p}<0.0001$ & $\mathrm{z}=4.301, \mathrm{p}<0.0083$ & $\mathrm{z}=4.144, \mathrm{p}<0.01$ & $\mathrm{z}=3.912, \mathrm{p}<0.0125$ & $\mathrm{z}=3.829, \mathrm{p}<0.0167$ \\
Satisfaction & $X^{2}=50.653, \mathrm{p}<0.0001$ & $\mathrm{z}=4.298, \mathrm{p}<0.0083$ & $\mathrm{z}=4.242, \mathrm{p}<0.01$ & $\mathrm{z}=3.611, \mathrm{p}<0.0125$ & $\mathrm{z}=4.048, \mathrm{p}<0.0167$ \\
Usefulness & $X^{2}=45.646, \mathrm{p}<0.0001$ & $\mathrm{z}=4.300, \mathrm{p}<0.0083$ & $\mathrm{z}=4.156, \mathrm{p}<0.01$ & $\mathrm{z}=3.385, \mathrm{p}<0.0125$ & $\mathrm{z}=3.758, \mathrm{p}<0.0167$
\end{tabular}

rent video see-through HMD solution has the advantage of being the least expensive and easiest to implement, disadvantages of the video see-through include a low resolution of reality, a limited fieldof-view, fixed focus distance and biocular display that can cause user discomfort, eye strain and fatigue. Finally, the test-bed system targets building blocks (LEGO), which is a restricted subset of general objects. The block structures were assumed to be in contact with the table horizontally during translation and rotation. The pose estimation problem was therefore constrained to three degrees of freedom (two degrees of freedom in translation and one degree of freedom in rotation) rather than six. The tracking algorithm needs to be improved to track objects of an arbitrary shape with six degrees of freedom [9]. It may additionally be possible to relax other constraints to support a wider variety of structures and open new research questions on AR-based context-aware visualization modes in unconstrained motion context.

\section{Conclusion}

This study evaluated the effectiveness of an AR-based contextaware assembly support system with different AR visualization modes proposed for object assembly, an AR display mode that display guidance information directly overlaid on the physical model, and an AR display mode in which guidance information is rendered on a virtual model adjacent to the real model. We found, somewhat surprisingly, that the visualization mode that renders guidance information on a virtual model separate from the physical model (Side-by-side mode) was preferable in every way to displaying such information directly overlaying the physical model. This result also held irrespective of whether we enabled a context-aware error detection mechanism. We believe that our results provide useful insight into the design of AR visualization techniques for assembly support systems.

\section{ACKNOWLEDGEMENTS}

This research was funded in part by Grant-in-Aid for Scientific Research (B), \#24300048 from the Japan Society for the Promotion of Science (JSPS), Japan, and by NSF CAREER award IIS-0845921, NSF awards IIS-0856045, and CCF-1012056.

\section{References}

[1] Arvika, http://www.arvika.de/.

[2] ARTESAS, http://www.mip.informatik.uni-kiel.de/tiki-index.php ?page $=$ artesas.

[3] R. Azuma. A survey of augmented reality. In Presence: Teleoperators and Virtual Environments, 6(4):355-385, 1997.

[4] F. Biocca, A. Tang, D. Lamas, J. Gregg, R. Brady, and P. Gai. How do users organize virtual tools around their body in immersive virtual and augmented environment?: An exploratory study of egocentric spatial mapping of virtual tools in the mobile infosphere. In Technical Report of Media Interface and Network Design Labs, Michigan State University, EastLansing, 2001.

[5] V. Buchmann, T. Nilsen, and M. Billinghurst. Interaction with partially transparent hands and objects. In Australasian Conference on User Interface - Volume 40, pages 17-20, 2005.

[6] J. Chen and B. MacIntyre. Uncertainty boundaries for complex objects in augmented reality. In IEEE Virtual Reality Conference, pages 247 $248,2008$.
[7] E. Coelho, B. MacIntyre, and S. Julier. Supporting interaction in augmented reality in the presence of uncertain spatial knowledge. In $A C M$ Symposium on User Interface Software and Technology, pages 111114,2005

[8] C. Furmanski, R. Azuma, and M. Daily. Augmented-reality visualizations guided by cognition: Perceptual heuristics for combining visible and obscured information. In International Symposium on Mixed and Augmented Reality, pages 215-320, 2002.

[9] A. Gupta, D. Fox, B. Curless, and M. Cohen. Duplotrack: a realtime system for authoring and guiding duplo block assembly. In $A C M$ symposium on User interface software and technology, pages 389402, 2012.

[10] R. Haines and T. Fischer, and E. Price. Head-up transition behavior of pilots with and without head-up display in simulated low-visibility approaches. In Technical Report of NASA Ames Research Center, Moffett Field, 1980.

[11] S. Henderson and S. Feiner. Augmented reality in the psychomotor phase of a procedural task. In International Symposium on Mixed and Augmented Reality, pages 191-200, 2011.

[12] S. Holm. A simple sequentially rejective multiple test procedure. In Scandinavian Journal of Statistics, 6:65-70, 1979.

[13] M. Livingston, J. Swan II, J. Gabbard, T. Höllerer, D. Hix, S. Julier, Y. Baillot, and D. Brown. Resolving multiple occluded layers in augmented reality. In International Symposium on Mixed and Augmented Reality, pages 56-65, 2003.

[14] A. Miller, B. White, E. Charbonneau, Z. Kanzler, and J. LaViola Jr. Interactive $3 \mathrm{~d}$ model acquisition and tracking of building block structures. IEEE Transactions on Visualization and Computer Graphics, 18(4):651-659, 2012.

[15] J. Molineros, V. Raghavan, and R. Sharma. Computer vision based augmented reality for guiding and evaluating assembly sequences. In IEEE Virtual Reality Annual International Symposium, page 214, 1998.

[16] U. Neumann and A. Majoros. Cognitive, performance, and systems issues for augmented reality applications in manufacturing and maintenance. In IEEE Virtual Reality Annual International Symposium, pages 4-11,1998.

[17] U. Neumann and A. Majoros. Cognitive, Performance, and Systems Issues for Augmented Reality Applications in Manufacturing and Maintenance. In IEEE Virtual Reality Annual International Symposium, 1998.

[18] C. Robertson, B. MacIntyre, and B. Walker. An evaluation of graphical context as a means for ameliorating the effects of registration error. IEEE Transactions on Visualization and Computer Graphics, 15(2):179-192, 2009.

[19] B. Schwald, and et al. Starmate: Using augmented reality technology for computer guided maintenance of complex mechanical elements. In E-work and E-commerce, pages 196-202, 2001.

[20] A. Tang, C. Owen, F. Biocca, and W. Mou. Comparative effectiveness of augmented reality in object assembly. In Annual SIGCHI Conference: Human Factors in Computing Systems, pages 73-80, 2003.

[21] D. Van Krevelen and R. Poelman. A survey of augmented reality technologies, applications and limitations. International Journal of Virtual Reality, 9(2):1-20, 2010. 